\title{
Ein virtuelles Gespräch zwischen Johann Götschl und Michael Muhr
}

\author{
J. Götschl
}

Online publiziert am 25. September 2012

(C) Springer-Verlag 2012

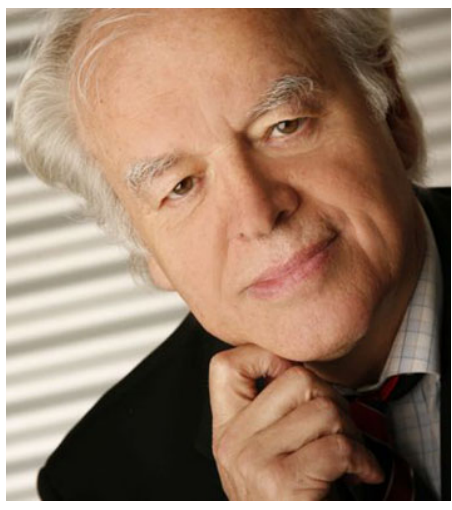

Hon. Prof. Univ.-Prof. Dr. Johann Götschl
Viele Begegnungen mit Michael Muhr haben in den vergangenen Jahren stattgefunden. Durch unsere gemeinsame Interessenslage haben wir viele Themenfelder behandelt und auch etliche offen lassen müssen. Insgesamt aber denke ich, die Grundkonturen des Denkens von Michael Muhr ganz gut verstanden zu haben. Natürlich liegt die volle Verantwortung für den folgenden Text, den ich

Michael Muhr zugeordnet habe, ausschließlich bei mir.

J. Götschl Lieber Michael, seit Jahren besprechen wir zentrale Themenfelder, die uns beide sehr bewegen und herausfordern. Wir interessieren uns sehr für entscheidende Charakteristika der weiteren Entwicklungen der wissenschaftlich-technologisch-industriellen Zivilisationsformen, für die demokratischen Grundlagen individueller und kollektiver Existenz und für die Zusammenhänge der Generationen. Darüber hinaus konnte ich mit Dir viele Problemfelder besprechen, wie das Netzwerk von wissenschaftlich-technologischer Kompetenz, gesellschaftlichen Werten und Offenheiten bis hin zu politischer Kompetenz für Bildung und Verantwortung. In unserem gemeinsamen Seminar zum Thema "Komplexität und Dynamik in der Wissensgesellschaft" wie auch in der Ethikkommission der TU Graz (CSIE) hast Du immer wieder herausgearbeitet und betont, wie wichtig es Dir ist, die Technologie im Allgemeinen, das technische Studium im Besonderen in neuere, integriertere und besser zu verstehende Perspektiven zu bringen. Worum geht es dabei für mittelfristiges Denken?

M. Muhr Es war und ist mir ein besonderes Anliegen, die wissenschaftlich-technologisch-industrielle Welt stärker in einen soziokulturellen wie auch sozioökonomischen Zusammenhang zu bringen, insbesondere auch in einen reflexiv-konstruktiven Zusammenhang. Die nach wie vor zu große Trennung - sicher zum Teil auch unbewusst - in technische und nicht-technische Lebensformen sollte zumindest mittelfristig überwunden werden können, wie wir dies ja gemeinsam in unserem Seminar gewissermaßen im Kleinen mit sehr starker interdisziplinärer und transdisziplinärer Ausrichtung vornehmen. Ähnliches gilt für Deine Vorlesung "Technik und Ethik", deren Einrichtung mir so wichtig war und die inzwischen hunderte Studenten absolviert haben. Ganz generell als kulturelle Grundorientierung geht es mir darum, wissenschaftlich-technologisch basiertes Denken und philosophischhumanwissenschaftliches Denken stärker zusammenzuführen. Oder anders ausgedrückt: Die Welt, in der wir alle leben, sollten wir integraler wahrnehmen bzw. uns ganzheitlicher aneignen. Denn die enorm ansteigende Komplexität und Dynamik unserer gegenwärtigen Gesellschaft lassen erkennen, wie eng die Chancen und Gefahren beieinander liegen. Bei den Versuchen, die Welt umfassender wahrzunehmen, um dadurch auch neue Gestaltungs- und Verwirklichungsmöglichkeiten zu finden, geht es mir weniger um spezifische Prioritäten in Bezug auf wissenschaftlich-technologisch und philosophisch-humanistische Lebensmodelle. Vielmehr geht es mir darum, das wissenschaftlich-technologische Denken philosophischhumanistisch und umgekehrt das philosophisch-humanistische Denken wissenschaftlich-technologisch zu durchdringen.

J. Götschl Viele Wissenschaftler, Forscher und Technologen gehen davon aus, dass wir Menschen uns auf immer mehr Grenzsituationen zubewegen. Da Komplexität und Dynamik der wissenschaftlich-technologischen Welt sowohl quantitativ als auch qualitativ ansteigen, werden auch die grundlegenden Bewertungen, Entscheidungen und Handlungen als existenzielle Weichenstellungen nicht nur dringender, sondern auch schwieriger. Vor diesem Hintergrund neigen Menschen auch dazu, anstelle vertiefter Analysen als Basis für Orientierung und Gestaltung zunehmend Zuflucht zu Pessimismus zu suchen. Von unseren Gesprächen weiß ich, dass Du weder Pessimist noch Zweckpessimist bist, sondern dass Du vielmehr auf der Suche bist nach den sich verändernden Bedingungen für Optimismus bzw. für Humanität. Wie ist Deine Sicht der Welt im Sinne eines erkenntnisorientierten und humanorientierten Optimismus?

M. Muhr Etwas - Du würdest es wahrscheinlich einen "humanistisch-begründbaren Optimismus" nennen - ist für mich eine zentrale und faszinierende Herausforderung, insbesondere auch, was die Zusammenhänge zwischen den Generationen betrifft. Das Menschenbild im Sinne eines "humanistischen Optimisten" ist eine Lebensform bzw. Weltsicht, in welcher sich zumindest drei Dimensionen zu einer einheitlicheren Lebenssicht zusammenfügen sollten. Erstens verstärkt sich die Erkenntnis dahingehend, dass Wissen, Können und Verantwortung wie nie zuvor zusammengehören, das heißt eigentlich, dass diese sich in spezifischer Weise untereinander bedingen. Zweitens vertieft sich die Erkenntnis dafür, dass die Differenzen zwischen Wissens- und Einkommensschichten gerechter zum Wohle aller gestaltet werden müssten, und drittens - eng mit diesen beiden Aspekten verbunden - ist es nötig, am Aufbau des sozialen und ethischen Zusammenhalts der modernen Gesellschaft verstärkt zu arbeiten. Wir haben es mit einer neuen Entwicklungsdy-

Götschl, Johann, Hon. Prof. Univ.-Prof. Dr., Karl Franzens Universität Graz und Technische Universität Graz, Merangasse 18, 8010 Graz, Österreich

(E-Mail: johann.goetschl@uni-graz.at) 
namik in Bezug auf Human- und Gefahrenpotentiale der modernen Gesellschaften zu tun, deren Typus sich rascher denn je erahnt über die Welt ausbreitet. Das Humanpotential in den wissenschaftlichtechnologisch-industriellen Gesellschaften ist etwas wie eine neuartige Kulturform, aus der heraus sich nur dann nachhaltige Vorteile realisieren werden, wenn der Zusammenhalt der Gesellschaft nicht von Individualismus oder gar Egoismus dominiert wird. Eine humane Kulturform wird von einem neuen Verhältnis von innerer und äußerer Freiheit der Individuen bestimmt sein müssen, in welchem individuelle Zuwächse an Freiheitsgraden besser der Idee eines Wohlfahrtsstaates entsprechen können. Ein innerer Zusammenhalt der Gesellschaft wird erst auf höherer Stufe möglich, wenn die dringend nötige gerechtere Verteilung von wissenschaftlich-technologischer Kompetenz, von ökonomischen Werten, von Anerkennung, Wertschätzung und Verantwortung verwirklicht werden.

J. Götschl Geht man davon aus, dass man wissenschaftlichtechnologische Kompetenz, Innovation, Wirtschaft und ethische Verantwortung in neue innere Zusammenhänge bringen will, dann stößt man sehr rasch auf Themenfelder wie Rationalität, Lernfähigkeit, Steuerbarkeit und Selbststeuerung von Gesellschaften. Man muss sich den Herausforderungen sehr schnell stellen - was gegenwärtig mit Recht immer mehr thematisiert wird - auf Grenzen der Steuerbarkeit insbesondere in Bezug auf wissenschaftlichtechnologisch-industrielle Prozesse wie auch in Bezug auf Staatsmodelle zu achten. Der Realitätsdruck ist offensichtlich enorm im Ansteigen, neue Beziehungen zwischen Rationalität einerseits und spezifischen Fantasien bis hin zu idealisierten esoterischen Denkformen andererseits zeigen sich, wobei vor allem letztere zum Teil auch als Fluchtaspekte oder auch als Verführungsaspekte fungieren können. Sind wir hier in neuer Weise gefordert, gleichsam bestimmte Aspek te der demokratischen Selbstorganisation auf den Prüfstand zu stellen, um konstruktive Weiterentwicklungen demokratischer Gesellschaften zu ermöglichen?

M. Muhr Wissen - insbesondere auch in Form von wissenschaftlicher Grundlagenforschung - stellt seit längerem für den Menschen etwas kategorial Neues und Einzigartiges dar, weil damit auch hohe Formen von Objektivität vorliegen und dadurch auch die Verständigung zwischen unterschiedlichen Gesellschaftsebenen und zwischen verschiedensten Kulturen verbessert wird. Man muss quasi im Gleichschritt daran arbeiten, die existenzielle Bedeutung von Wissenschaft und Technologie besser zu verstehen, um die äußerst sensitiven Zusammenhänge bzw. Interdependenzen zwischen der Eigendynamik und der Steuerung, das heißt des Eingriffs in die Eigendynamik, expliziter zu machen. Wir haben offensichtlich zuwenig gut begründetes Steuerungswissen, in dem sich die Einheit von Erkenntnis und Humanität theoretisch und praktisch manifestiert. Im Kern bedeutet dies, dass es zur Anhebung von Kompetenz und Verantwortung sowohl in gesellschaftlichen Prozessen wie im Besonderen auch in den legistisch-politischen Ebenen kommen muss. Hierin liegt eine zentrale Herausforderung für die mittlere Zukunft, da sowohl eine Anhebung als auch eine Weiterentwicklung von demokratischen Grundformen erforderlich sein wird. Denn wissenschaftlichtechnologisch-industrielle Wirkungen auf die Mikro- und Makrostrukturen der gegenwärtigen Gesellschaft nehmen dramatisch zu, was vor allem auch einen Zuwachs an qualitativer Urteilsbildung und damit an qualitativen Elementen von Demokratie erforderlich macht. Vor diesem Hintergrund ist evident, dass die Verstärkung aller kooperativen Lebensprozesse als Ausdruck des inneren Zusammen- halts einer Gesellschaft von autoritären oder gar totalitären Denkund Lebensformen wegführt.

J. Götschl Um von größeren Aspekten zu scheinbar kleineren Aspekten zu gelangen, gibt es die bekannten Diskussionen um die Einführung von Studiengebühren mit offensichtlich nicht sehr hohem Konsensniveau. Dies heißt ja eigentlich - was vielleicht befruchtend werden könnte - dass es zu einem viel tieferen Durchdenken der Zusammenhänge zwischen Bildung, Ausbildung und Ökonomie kommen muss. Man befindet sich zum Teil in weltanschaulichen Differenzen und zum Teil auch in ökonomischen Zwängen. Offensichtlich verstärkt sich in einem erkennbar größeren Ausmaß die Tendenz zur Ökonomisierung von Wissen und Bildung. Diese Ökonomisierung bzw. Monetarisierung von Wissen - so wird Wissen oft als neuer Rohstoff des 21. Jahrhunderts propagiert - wird besonders stark auch auf die Wissenschaft bezogen. Liegt in diesem Prozess vielleicht eine noch zu verborgene Veränderung des Menschenbzw. Gesellschaftsbildes? Ist der "Homo Oeconomicus" das zentrale Paradigma für das 21. Jahrhundert?

M. Muhr Die öffentliche Diskussion um die Studiengebühren ist ein wichtiges Symptom wie auch ein soziales Oberflächenphänomen besonderer Art. Denn dahinter steckt die wahrscheinlich noch lange nicht hinreichend wahrgenommene und verstandene neue soziale Wirklichkeit. Denn Wissen - insbesondere wissenschaftliches Wissen, wissenschaftlich basierte Technologie und technologiebasierte Wissenschaft - hat zweifellos kulturübergreifende Bedeutung für das Menschsein. Die vielleicht nicht hinreichende Wahrnehmung der Ökonomisierung von Wissen verschiebt dessen existenzielle Bedeutung. Denn zumindest eine zu frühe Ökonomisierung der Wissenserzeugung und der Wissensverbreitung wird mitbestimmend sein für das Generieren, das Finden wie auch für die Schwerpunktorientierungen und Schwerpunktsetzungen. Obwohl eine sensitiv differenzierte Ökonomisierung von Wissen nicht grundsätzlich negativ sein muss, so sind wir doch weit davon entfernt, die Möglichkeiten und Grenzen einer Ökonomisierung bzw. Monetarisierung der Ursprünge von Kreativität und Innovation, von Motivation und Interessen für Mensch und Welt zu kennen. Hier sehe ich zumindest zwei Gefahrenpotentiale. Erstens: Wissenschaftliche Wissensformen dürfen nur zum Teil ökonomisch „instrumentalisiert” werden, weil der zentrale Wert wissenschaftlich-basierter Rationalität in der selbsterkennenden Sinnbestimmung für Individuum und Gesellschaft liegt. Wird die ökonomische Funktionalisierung und Instrumentalisierung in Bezug auf Grenzen zu wenig beachtet, wird es zu Humanverlusten kommen. Zweitens: Eine nicht sensitiv differenzierte Ökonomisierung des Wissens - bei der die Grenzen erst durch intensive Forschung genauer ausgelotet werden müssten verändert das Verhältnis zwischen pragmatischen und reflexiven Kategorien menschlicher Existenz. Denn im Denken der Gegenwart scheint sich die Richtung hin zu Pragmatismus und Funktionalismus zu verstärken. Die damit verbundenen Erfolge sind eben nur die eine Facette des Menschseins. Denn was Menschsein grundlegend benötigt, ist ein dynamisches Gleichgewicht zwischen pragmatischer und reflexiver Rationalität. Dies sind einige Aspekte dafür, dass Studiengebühren wahrscheinlich ein zentrales Thema für das nächste Jahrzehnt sein werden. Denn die sozio-kulturellen Bedingungen für Entstehung bzw. Verteilung von Ideen, Innovationen, Interessen, Begabungen und Verantwortlichkeiten korrelieren - jedenfalls was den gegenwärtigen Stand höherer Wissensansprüche betrifft - nicht hinreichend adäquat mit den ökonomisch-monetären Steuerungsprozessen. Ich denke, wir sollten uns mehr denn je der gewaltigen Herausforderung einer Problemlösung stellen, wodurch 
die Grenzen und Möglichkeiten genauer identifiziert werden sollten, um den Wert oder den Unwert bestimmter Zusammenhänge zwischen Wissen und Geld als Ausdruck gesuchter Humanitätsorientierung expliziter zu gestalten.
J. Götschl Lieber Freund und lieber geschätzter Wissenschaftskollege, ich verstehe dies auch als Einladung an mich, Problemanalysen so schnell wie möglich aufzunehmen. Es ist einfach schön und würdig, wenn man auf solche Weise gewollt wird. 